\title{
El estigma en la persona adicta
}

\section{Stigma in the Addicted Person}

\author{
Mireia Pascual Mollá*,**, Francisco Pascual Pastor********. \\ *Responsable de prensa de SOCIDROGALCOHOL. **Directora campaña \#rompeleestigma. \\ ***Coordinador UCA Alcoi. ****Presidente SOCIDROGALCOHOL.
}

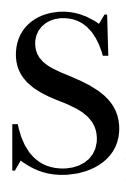
eñal, marca, huella, vestigio, signo, llaga, baldón, afrenta, mancha, mancilla o sambenito, son algunos de los sinónimos que utilizamos en castellano para acercarnos a la compresión de la palabra estigma que por otro lado viene definida por la Real Academia de la lengua española como marca o señal en el cuerpo, pero también como desdoro, afrenta o mala fama.

Ha habido a lo largo de la historia múltiples enfermedades, daños o alteraciones que han sido catalogadas de esta forma y que lo único que han conseguido ha sido apartar a la persona de su entorno social, de poder recibir el apoyo necesario y de disfrutar de sus derechos humanos y finalmente ha terminado siendo menospreciado, marginado, alguien a quien debía evitarse. Se trata, por lo tanto, de un atributo profundamente desacreditador.

Esto lo vimos en enfermedades como la lepra, la peste, la esquizofrenia e incluso la epilepsia. ¿Qué sucede? ¿Estos individuos no son personas que merecen un trato igual al resto?

Erving Goffman definió el "estigma" como una expectativa de un descrédito. Juicio de uno mismo por otros en un contexto particular (Goffman, 1968)

En las últimas décadas, además de la estigmatización de los enfermos mentales, hemos visto como se ha producido el mismo fenómeno en el VIH/ SIDA, por el propio síndrome o incluso por las características personales de quien lo sufre. Y aunque no es reciente, las personas que tienen un trastorno por uso de sustancias, con todo lo que conlleva, son víctimas de este fenómeno. Lo que, por otro lado, no deja en muy buen lugar a la propia sociedad.

Y es que además en un determinado momento había un binomio VIH / Drogas que contribuía aún más a este pre- juicio. La sociedad tiende a crear cajones donde clasifica y mete a ciertos individuos y los medios de comunicación facilitan estas representaciones y creencias. (Rengel, 2005). Además, hay una tendencia a etiquetar de forma negativa a quien padece un trastorno adictivo, resaltando la parte negativa y reseñando su condición como parte importante de la noticia, aun cuando no lo sea en ese caso.

Según el sociólogo Javier Rubio, el proceso de la construcción de este estigma es siempre arbitrario, cultural y surge de la necesidad de censurar a aquellas personas que se desvían de lo que es o no asumido como aceptable desde el punto de vista social y cultural. Opera como una definición totalizadora con capacidad para desacreditar a la persona/consumidora de sustancias tóxicas en el intercambio social. El proceso de estigmatización convierte al drogodependiente en un ser desposeído, su definición social se establece por comparación con los no consumidores y esta comparación sirve para fijar su posición social como alguien que es diferente e inferior. Y es que además el propio consumo/adicción le genera un deterioro del entorno social (de familiares, iguales, conocidos, vecinos, etc.) y laboral, al vivir el adicto la vida a través de la sustancia, creándose un estilo de vida psicosocial acorde con su nueva situación y su nuevo rol (drogodependiente) (Rubio, 2001).

El consumo de alcohol y otras drogas hacen que la persona tenga conductas no adecuadas al constructo social, pueden padecer enfermedades físicas y/o psíquicas que les alejan del contexto de normalidad, presentan en ocasiones actuaciones delictivas y se les relaciona, con accidentabilidad, malos tratos o delincuencia. Calificándolos, de viciosos, pasotas o delincuentes, sin tener en cuenta su condición de persona que ha perdido el control sobre sus actos o la necesi-

Recibido: Julio 2017; Aceptado: Septiembre 2017.

Enviar correspondencia a:

Mireia Pascual Mollà. Grupo de Alcohólicos Rehabilitados de Alcoi. C/ El Camí, 40. (bajos) 03801. Alcoi.

Mail: mireia.pascual@gmail.com 
dad imperiosa de consumo por la propia adicción a la sustancia. Aunque en principio el estigma es menor para los consumidores de drogas legales, cuando se pasan ciertos umbrales y consecuencias del consumo esta percepción se desvanece. Como podemos observar con el consumo de alcohol entre los sin techo y el riesgo de exclusión social que presentan estas personas (Panadero, Vázquez y Martín 2017).

El empeño de ciertos colectivos por desestigmatizar algunas cuestiones de género, las enfermedades mentales o el VIH, puede servirnos de ejemplo y guía para intentar romper con el estigma del adicto.

Se entiende que una carga pesada de simbolismo rodea el uso de sustancias psicoactivas. Las sustancias psicoactivas pueden ser productos de prestigio, pero uno u otro aspecto de su uso parece atraer el estigma y la marginación casi universales. Estableciendo incluso una relación del consumo de sustancias y el crimen (Esbec y Echeburúa, 2016).

Los procesos de estigmatización incluyen el proceso íntimo de control social entre familiares y amigos; decisiones sociales y de salud y las decisiones políticas gubernamentales. Lo que es negativamente moralizado comúnmente incluye incurrir en salud, accidentes o problemas sociales, intoxicación, adicción o dependencia, así como la pérdida de control. Ahora a esto lo denominamos Trastorno por Uso de Sustancias. La marginación y el estigma comúnmente se suman a este efecto. Aquellos que están en tratamiento por problemas de alcohol o drogas son marginados frecuentemente y desproporcionadamente, lo que produce unos resultados, adversos, negativos en el proceso terapéutico. (Room, 2005)

Se trataría de evitar la falta de comprensión hacia personas con trastorno adictivo ya que la gran mayoría de las personas con problemas de adicción sufren el rechazo social y son apartados y estigmatizados.

Como en una paradoja, vemos que en relación con ciertas sustancias los consumos son alentados, publicitados y aplaudidos pero las consecuencias rechazadas.

Observamos tres vertientes en este problema,

a. El autoestigma, u ocultamiento del problema por parte de quien lo padece (aislamiento, no reconocimiento...), muchas veces condicionado por la vergüenza lo que afecta a los comportamientos de salud y genera invisibilidad por lo que repercute negativamente en la toma de decisiones socio-políticas (Dolezal y Lyons, 2017).

Por ejemplo, el autoestigma ocurre cuando las personas con enfermedades mentales internalizan los estereotipos negativos y los prejuicios sobre su condición y ello puede reducir el comportamiento de búsqueda de ayuda y la adherencia al tratamiento. El problema es que se ha intentado luchar contra el autoestigma y la eficacia de las intervenciones, de momento es incierta, según un metaanálisis realizado en 2013 (Büchter y Messe, 2017).
Al final la propia concepción o la autopercepción es la de "Soy inferior. Por lo tanto, la gente me tendrá aversión y yo no me sentiré seguro con ellos" (Perry, Gawel y Gibbon, 1956).

b. El estigma social por rechazo por parte de la población que no entienden lo que es una persona con una adicción y siguen catalogando de vicio la adicción a cualquier sustancia. Se han intentado valorar distintas actuaciones con enfermos mentales y los resultados ponen de relieve la importancia de focalizar los resultados conductuales del proceso de estigmatización (discriminación e inequidad social), lo cual es consistente con los modelos de justicia social o de derechos que enfatizan la equidad social y económica para las personas con discapacidades (como acceso equitativo a servicios, educación, trabajo, etc.). No obstante, también ponen en tela de juicio los grandes enfoques de educación pública en favor de intervenciones más específicas basadas en el contacto (Stuart, 2016), en ese sentido el programa propuesto por Socidrogalcohol, cuenta desde un primer momento con los colectivos de personas y familiares afectados. Y, por último,

c. El estigma en el sector sociosanitario que ven molestos a este tipo de personas con tendencia a etiquetarlos antes incluso de escucharlos. Probablemente la falta de sensibilización o incluso de formación curricular provoca entre los profesionales cierto rechazo hacia estas personas.

$Y$ sin añadir nuevos escenarios, no olvidemos que el estigma y sus consecuencias se incrementan cuando se trata de una mujer o si el adicto tiene alguna enfermedad física y sobre todo mental asociada, es decir, cuando padece una comorbilidad o coexistencia de distintas alteraciones, enfermedades o trastornos. (Foundation Transform Drug Policy, 2010)

En cierta forma esta problemática se palió al ser considerados los adictos como enfermos, según la definición de enfermedad de la OMS, como la "Alteración o desviación del estado fisiológico en una o varias partes del cuerpo, por causas en general conocidas, manifestada por síntomas y signos característicos, y cuya evolución es más o menos previsible" ya que para la OMS la Salud es un estado de completo bienestar físico, mental y social, y no solamente la ausencia de afecciones o enfermedades (OMS,1946) e incluso bajo la consideración de trastorno por dependencia, lo que hizo que estas personas tuvieran el mismo derecho asistencial que cualquier otro tipo de enfermo. Lo que ha permitido que se haya dado respuesta asistencial a más personas, aunque esto finalmente no se ha producido en toda su extensión.

Pero lo importante quizá se fundamenta en el concepto de salud como el estado de pleno bienestar físico, mental y social, y no solamente la ausencia de enfermedad. Por tanto, 
no solamente cuenta el buen estado físico o fisiológico, sino también los aspectos psicológicos y cómo influye el entorno (socioeconómico, familiar, laboral, emocional, medioambiental), que al fin y al cabo es lo que se debe considerar en cualquier persona con un trastorno adictivo desde el modelo bio-psico-social, imperante en la atención a estas personas.

Hay que tener en cuenta que los recursos asistenciales para atender a estas personas también refuerzan en muchas ocasiones este estigma y las administraciones en general olvidan la importancia de invertir en la recuperación de estas personas, desconocen que el ahorro que podría producirse al invertir en prevención es mucho mayor que el coste y es que todas las dimensiones: las sociales, asistenciales, la prevención, los recursos administrativos, todos, están impregnados por el estigma.

La estigmatización de las familias que apoyan a un miembro adulto con abuso de sustancias es común y socava su capacidad para apoyar a la persona y mantener su propio bienestar. En un estudio reciente el "mantener el secreto y minimizar el contacto con los demás" y "falta de conocimiento y empatía" complican el problema y se habla del papel útil de la enfermería y, en general, de los profesionales que trabajan en adicciones que con un enfoque especial podrían ayudar a reducir el estigma, desafiando las actitudes de algunos clínicos y mejorando las maneras de comunicarse con las familias. (McCann y Lubman, 2017)

La sociedad científica Socidrogalcohol al corriente de este hecho plantea un plan de sensibilización con el objetivo de reducir la estigmatización de las personas con trastornos adictivos, con un programa que plantea los siguientes objetivos:

- Favorecer el abordaje integral, público y gratuito, asumiendo la coordinación con Organizaciones No Gubernamentales, organizaciones de pacientes y la iniciativa privada, en el que tengan igual importancia los aspectos físicos, psíquicos y sociales para lograr la total recuperación de la persona.

- Reclamar la existencia de estructuras asistenciales acordes a las necesidades de los pacientes y sus familias: Tratamientos ambulatorios, Recursos hospitalarios para desintoxicación, Centros de día, Comunidades Terapéuticas, programas específicos de trastornos duales, Viviendas tutelada, Programas de reinserción y Apoyo y coordinación con los grupos de ayuda mutua.

- Normalizar los servicios y recursos asistenciales: Normalización de los recursos integrados dentro de las redes públicas de salud.

- Dotar cada servicio con unos equipos mínimos de profesionales, acordes a sus características, en los que se trabaje de forma multidisciplinar con un enfoque bio-psico-social.

- Reclamar y reforzar los derechos de estos pacientes para que reciban un trato y una atención como cualquier paciente que pueda sufrir otra patología.
- Exigir el respaldo por parte de la administración a las organizaciones de pacientes y familiares

- Aumentar el conocimiento social sobre que la adicción es una enfermedad

- Aumentar la conciencia social acerca de la necesidad de reinserción de las personas que la sufren y de que el estigma no contribuye a ello, sino que lo complica.

Para ello, se ha diseñado un programa de actuación en el que se han tenido en cuenta las tres vertientes del problema: la propia persona, al contar con los colectivos de adictos y sus familiares; la sociedad; y los profesionales sociosanitarios. Para ello los contenidos y materiales se han elaborado teniendo en cuenta estos actores. Por un lado, se ha redactado un documento que contiene los puntos de equidad, homogeneidad y derecho a la asistencia antes expuestos y se han solicitado adhesiones al mismo para sensibilizar, pero, sobre todo, para dar la máxima difusión a todo el programa y que otros sientan que también forman parte del proceso. Hemos podido contar con asociaciones de pacientes, colectivos de profesionales, fundaciones, etc... tanto de España como de distintos puntos de Europa.

Al mismo tiempo se ha realizado un material escrito, con dípticos y folletos, que se ha complementado con una serie de carteles específicos en los que se da al adicto el mismo rango que a otra persona que precise atención socio-sanitaria, todo ello acompañado de gifs, vídeos de testimonios para redes sociales, spots de TV y cuñas de radio. El objetivo es que el mensaje se difunda al máximo de público posible. Y por último se está elaborando material para poder llegar también a sensibilizar a todos los profesionales, contando con la ayuda de enfermería, colegios profesionales, de médicos, psicólogos y trabajadores sociales. Se hacen servir los medios de comunicación y, además, se organizan debates por autonomías y a nivel nacional para tener el máximo eco posible.

Además, no podíamos dejar de lado las redes sociales. La campaña cuenta con el hastag \#RompeElEstigma que se utiliza tanto en Facebook como en Twitter para difundir y recalcar el mensaje, y en muchas ocasiones utilizamos un segundo hastag \#CombateLaAdiccion. La elección del nombre de la campaña y del hastag, que es el mismo, se ha hecho por una sencilla razón, el estigma, como hemos comentado al inicio de este artículo, es algo que viene acompañando multitud de enfermedades, utilizar el mismo mensaje nos permite unir fuerzas con otros colectivos que también precisan de esa lucha. Aunque en ocasiones nos interese focalizar para hacer llegar el mensaje de que el estigma está presente en las adicciones, y por eso, aparece también siempre la palabra 'adicción'.

Los materiales producidos trasmiten dos mensajes principales. 1. Que la adicción es una enfermedad. 2. Que cualquiera puede sufrirla y que su aspecto, edad, sexo, condición, etc. no puede servir para etiquetar a la persona. Para ello, los materiales elaborados son una serie que se com- 
plementa, es decir, las cuñas de radio, el spot, los carteles y los dípticos son una serie que utiliza los mismos personajes, los mismos mensajes y los mimos escenarios.

Para identificar la campaña, hemos dibujado una mascota, en forma de pájaro, que, enjaulado en su estigma, logra liberarse de él y conseguir su libertad. Un dibujo simpático y desenfadado que sin dramatizar intenta llegar y ser punto de unión y comprensión para todos en este hándicap para la correcta atención a las adicciones. El propio pájaro evoluciona junto con la campaña enfrentando su propio 'autoestigma', por lo que el escudo que le caracteriza cuenta al principio con una ' $\mathrm{E}$ ' de 'Estigma' y acaba por arrancárselo para que debajo aparezca el escudo con la 'L' de 'Libertad'.

Para difundir la campaña, además de las acciones descritas con anterioridad se ha aprovechado la estructura y organización por delegaciones autonómicas de Socidrogalcohol. Tras la impresión del material, se ha realizado una distribución por autonomías y cada una de las delegaciones han sido las encargadas de distribuir a los distintos recursos. Además se anima a que las adhesiones al documento presentado en primer lugar, utilicen y sean parte activa también de esa distribución, con la opción de añadir una coletilla, al final por ejemplo de las cuñas, en las que se añada: 'Colabora...y el nombre de la asociación o entidad'. Con esto se pretende sumar, entendemos que esta tarea desestigmatizadora es de todos y de cada uno de los agentes sociales involucrados, sino difícilmente llegaremos a la sociedad general.

La campaña no está cerrada sino más bien al contrario, está viva y dotada de un cambio continuado que se va adaptando a la sociedad y a los momentos, así como a las observaciones de los que puedan ponerla en práctica. Saldrán seguro, nuevos materiales en múltiples formas, vídeos, publicaciones, etc. Cuanto menos debemos conseguir plantear la palabra estigma dentro de la problemática de los trastornos adictivos, para por lo menos animar a la reflexión y debate, porque sin esto no podremos contribuir ni animar al cambio o al planteamiento de cómo afrontarlo.

La importancia de la realización de este programa contra el estigma radica según palabras las palabras de Ban Ki-moon, Secretario general de la ONU en 2008 (Ki-moon, 2008).

"Nadie debe ser estigmatizado o discriminado debido a su dependencia a las drogas. Pido a los gobiernos asiáticos enmendar leyes penales anticuadas que criminalizan a los sectores más vulnerables de la sociedad, y tomar todas las medidas necesarias para asegurar que estas personas vivan dignamente."

\section{Bibliografía}

Büchter, R.B. y Messe, M. (2017). Interventions for reducing self-stigma in people with mental illnesses: a systematic review of randomized controlled trials. German Medical Science, 15, Doc07. doi:10.3205/000248.

Dolezal, L. y Lyons, B. (2017). Health-related shame: an affective determinant of health? Medical Humanities, pii: medhum-2017-011186. doi:10.1136/medhum-2017-011186.

Esbec, E. y Echeburúa, E. (2016). Substance abuse and crime: considerations for a comprehensive forensic assessment. Adicciones, 28, 48-56. doi:10.20882/adicciones.790.

Goffman, E. (1968). Stigma: Notes on the Management of Spoiled Identity. New York: Simon and Schuster.

Foundation Transform Drug Policy, (2010). Recuperado de http://www.countthecosts.org/sites/default/files/Stigma_Spanish.pdf.

Ki-moon, B. (2008). Mensaje del 26 de Junio de 2008. En el 60 aniversario de la declaración Universal de los Derechos Humanos. Recuperado de http://www.un.org/es/ sections/universal-declaration/60th-anniversary-universal-declaration-human-rights-1948-2008/index.html.

McCann, T.V. y Lubman, D.I. (2017). Stigma experience of families supporting an adult member with substance misuse. International Journal of Mental Health Nursing. Avance de publicación on-line. doi:10.1111/inm.12355.

Organización Mundial de la Salud. (1946). Acta constituyente de la Organización Mundial de la Salud. Recuperado de http://www.who.int/suggestions/faq/es/.

Panadero, S., Vázquez, J.J. y Martín, R.M. (2017). Alcohol, poverty and social exclusion: Alcohol consumption among the homeless and those at risk of social exclusion in Madrid. Adicciones, 29, 33-36. doi:10.20882/adicciones.830.

Perry, H. S., Gawel M. L. y Gibbon, M. (1956). Clinical Studies in Psychiatry. Nueva York: W. W. Norton \& Company.

Rengel Morales, D. (2005). La construcción social del 'otro'. Estigma, prejuicio e identidad en drogodependientes y enfermos de sida. Gazeta de Antropología, 21, artículo 25.

Room R. (2005). Stigma, social inequality and alcohol and drug use. Drug and Alcohol Review, 24, 143-155.

Rubio Arribas, J. (2001). Proceso de la construcción social de un enigma: La exclusión social e drogodependiente. Nómadas, 4. Recuperado de http:/ /www.redalyc.org/articulo.oa?id=18100414.

Sturat, H. (2016). Reducing the stigma of mental illness. Global Mental Health (Cambridge), 3, e17. doi:10.1017/ gmh.2016.11.

\section{Conflicto de interés}

Los autores declaran que no existe ningún conflicto de interés. 